\title{
Complex Network Characteristics and Team Performance in the Game of Cricket
}

\author{
Rudra M. Tripathy ${ }^{1}$, Amitabha Bagchi ${ }^{2}$, and Mona Jain ${ }^{2}$ \\ 1 Silicon Institute of Technology, Bhubaneshwar, India \\ ${ }^{2}$ Indian Institute of Technology, Delhi, India
}

\begin{abstract}
In this paper a complex network model is used to analyze the game of cricket. The nodes of this network are individual players and edges are placed between players who have scored runs in partnership. Results of these complex network models based on partnership are compared with performance of teams. Our study examines Test cricket, One Day Internationals (ODIs) and T20 cricket matches of the Indian Premier League (IPL). We find that complex network properties: average degree, average strength and average clustering coefficient are directly related to the performance (win over loss ratio) of the teams, i.e., teams having higher connectivity and well-interconnected groups perform better in Test matches but not in ODIs and IPL. For our purpose, the basic difference between different forms of the game is duration of the game: Test cricket is played for 5-days, One day cricket is played only for a single day and T20 is played only for 20 overs in an inning. In this regard, we make a clear distinction in social network properties between the Test, One day, and T20 cricket networks by finding relationships between average weight with their end point's degrees. We know that performance of teams varies with time - for example West Indies, who had established themselves as the best team during 1970s now is one of the worst teams in terms of results. So we have looked at evolution of team's performances with respect to their network properties for every decade. We have observed that, the average degree and average clustering coefficient follow similar trends as the performance of the team in Test cricket but not in One day cricket and T20. So partnership actually plays a more significant role in team performance in Test cricket as compared to One day cricket and T20 cricket.
\end{abstract}

Keywords: Complex Networks, Social Networks, Cricket, Partnership.

\section{Introduction}

Networks or graphs provide a representation of highly complex phenomena in a simpler form allowing us to understand the system in a better way. Obviously, the applicability of this approach depends on the ability to identify meaningful interacting units and the relationships connecting them. These complex networks are analyzed in terms of some static properties based on degree and connectivity. Cricket is probably the only sport where a dyadic relationship exists among a 
group of players in the form of batting partnership (runs scored between two players together). Out of 11 players of a batting team only two are batting at a given time. So it is quite natural to think of cricket as a dyadic social network. It is common wisdom that batting partnerships, sometimes called "offensive relationships", play a significant role in performance (win over loss ratio) of the team in cricket. In order to put this wisdom on a rigorous basis we modeled cricket as a social network based on partnership and studied the network properties of the cricket network. Our main observation is that team performance in Test cricket definitely depends on the presence of players who form many strong partnerships, but in the shorter forms of the game this effect is weakened. While this result is what we might expect, our contribution is methodological in that we describe a set of metrics by which this may be established.

Experiments and overview of results: Here a weighted network model has been considered to represent each team where each cricket player is a node in the network. Two nodes are connected by an edge if they have scored runs in partnership and the weight of the edge is given by the ratio between total number of runs scored and number of partnerships. Different social network metrics are then applied on the created networks to get insight into the nature of social relationships between players of a team and the effect of these relationships over the performance of a team. Some of others interesting observations from the cricket network are as follows:

- Degree distribution as well as strength distribution follows power law [1|2 3 4 45] and it also demonstrates the small world phenomena 6773.

- There are few hubs in the partnership graphs. They represent popular (higher degree) batsmen of their respective countries like Sachin Tendulkar and Ricky Ponting.

- In the cricket network, clustering coefficient of nodes decreases with their degrees. This behavior is explained as the larger-degree players partner with players who were on the team in different time periods.

- The cricket network satisfies disassortative properties. So in the cricket network, higher degree nodes have majority of neighbors with lower degrees, whereas opposite holds for low-degree vertices

- We found that clustering coefficient and average degree are directly related to the performance of the team, i.e., a team having higher connectivity and well-interconnected groups performs better.

- We show that partnership plays a more significant role in Test cricket than One day and T20 for team performance.

Dataset Description: The partnership data were collected from the StatsGuru utility on the ESPNCricinfo website 8. Advance filter tab was used to obtain the overall partnership summary of all the Test matches played between $1^{\text {st }}$ Jan. 1950 to $31^{\text {st }}$ Dec. 2008 and One day matches played between $1^{\text {st }}$ Jan. 1971 to $15^{\text {th }}$ July 2009 by each of the desired team. We have considered only those partnerships between two players who have scored more than 30 
runs in three such matches in Test cricket and scored more than 20 runs in five such matches in One day cricket. The reasons for taking different measures for Test and One day cricket are - One day cricket is played only for a single day, whereas Test cricket is played for five days and the number of One day matches played in a calendar year is more than that of Test matches. For the shortest format of the cricket, i.e., T20, we have used Indian Premier League (IPL) data. The Cricinfo website is used to gather the partnership data for all six seasons in which IPL tournament has been played, i.e., from 2008 to 2013. Each of the team in IPL has nearly played 100 matches. Therefore, we have not kept any restriction on the minimum partnership score or minimum number of matches for the IPL data. Some part of our analysis was done in 2010, particularly for the One day and Test cricket. Hence for Test and One day cricket we have used the old crawled dataset.

The remainder of the paper is structured as follows: Section 2 contains related works, Section 3 deals with background and motivation for this work, Section 4 we discuss how to model cricket as a complex network, Section 5 we relate the properties of cricket network with respect to the performance of the corresponding team and finally in Section [6 we draw some conclusions and highlight future work.

\section{Related Work}

Large complex systems, such as biological, social, technological and communication systems, have been analyzed using network models. The Internet [5], the World Wide Web [1], the online social networking services [2], the scientific collaboration networks (SCN) 4] are just to name a few. A number of studies have been done to investigate the topological characteristics of many networks. Milgram's work [7] giving concepts like six degrees of separation and small world are the most popular in this area. Measures such as degree of a node, average nearest neighborhood degree and clustering coefficient have been well studied for the unweighted network 9. Few studies have also been done in weighted networks. In 10, Newman proposed that properties of weighted networks could be studied by mapping them to unweighted multigraphs. In [11, Barrat et al. have given a quantitative and general approach to understand the complex architecture of real weighted networks. The analysis of team performance of sports leagues using complex network properties can be found in the literature. Vaz De Melo et. al. 12 have analyzed the National Basketball Association (NBA) database using complex network metrics, such as clustering coefficient and node degree. They have shown that the NBA network can be characterized as a small-world network and the degree distribution of players follows a power law. They have also proposed models for predicting the behavior of a team based on box-score statistics and complex network metrics. Results showed that only the box-score statistics cannot predict the correct behavior. But a mixed model (the network properties together with the box score statistics) can give more accurate results. In another study, Onody and De Castro [13] studied the Brazilian National Soccer Championship statistics from 1971 to 2002 by forming a bipartite network 
between the players and the clubs and they found that the connectivity of players had increased over the years while the clustering coefficient declined. This means, the players' professional life is growing longer and the players' transfer rate between clubs is going up. One of the major studies in the game of cricket is done by Uday Damodaran. He analyzed the ODI batting performance of Indian players from 1989 to 2005, using stochastic dominance rules [14. His study includes 4 specialist batsmen and 1 bowler and the results found were intuitive, such as Sachin Tendulkar proved to out-stand other players while Rahul Dravid dominated others till 20 overs. In their work [15, Allsopp and Clarke have rated cricket teams using multiple linear regression for both One day and Test. They have found Australia and South Africa are more rated teams than others. In this work, we have modeled cricket as a complex network and analyzed cricket using complex network properties. In our opinion similar work is not present in the literature. This is the first time that the game of cricket has been put into a social network framework.

\section{Introduction to Cricket}

The game of cricket originated in England. The game is played between two teams of eleven players each. The teams comprises some batsmen, some bowlers and a wicket-keeper. There are three forms of the game in cricket: Test matches, ODIs and T20. Test match cricket is the longest form of match, and is played over five days, with three sessions of two hours on each day. The first Test match was played between England and Australia in 1877 and since then a total of 1920 matches have been played up to $20^{t h}$ July 2009. A One day or limited-over match lasts for only a single day, where each team bats only once and their innings are limited to a fixed number of overs, usually fifty. The first One day international was played in Melbourne, Australia, in 1971, since then total number of 2861 number of matches have been played. One day cricket is more popular than Test cricket because of aggressive, risky and entertaining batting, which often results in a close finish. T20 is the shortest type of cricket as only 20 overs are played per inning. Indian Premier League(IPL) is a league for T20 championship in India. Both Indian and International players take part in the tournament. The first season of IPL was played in 2008 involving a total of 8 teams. Since then six IPL tournaments have been played.

Partnership In cricket two batsmen always bat together which is called partnership. Only one batsman is on strike at any time. If one of them got out then the partnership is broken. The measures which are used to describe a partnership are the number of runs scored, the duration of the partnership and the number of balls faced. Partnership plays a key role in match outcome in Test cricket as well as in One day cricket.

\subsection{Motivation}

As we know that the performance of a team for a particular period is measured by the number of matches they win in that period. But perhaps the better 


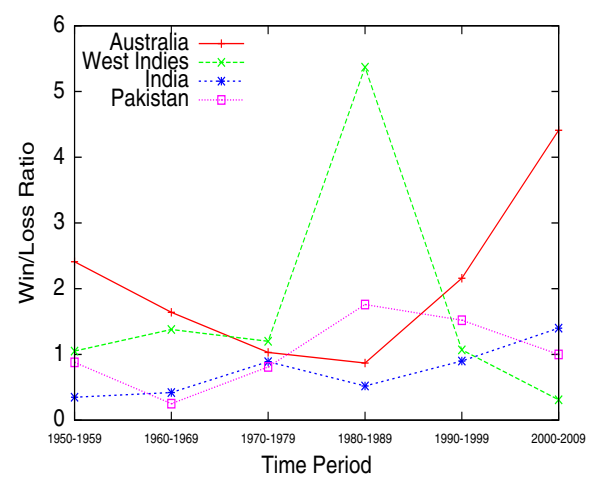

(a) For Test

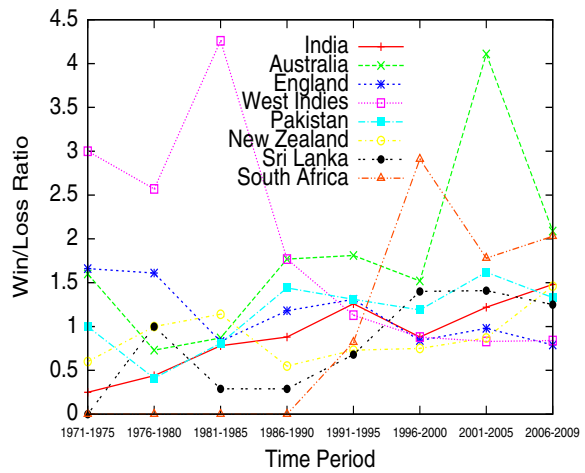

(b) For One day

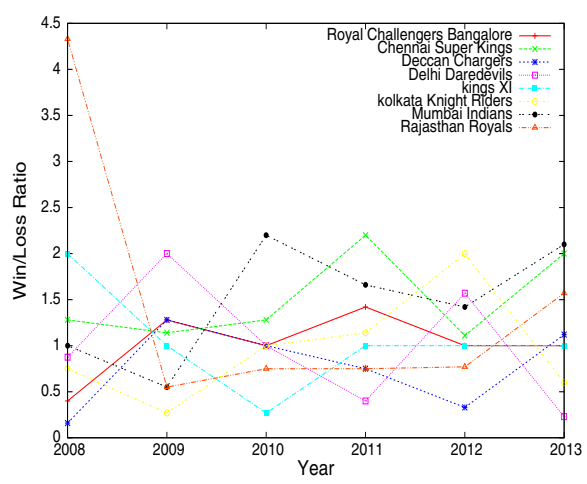

(c) For IPL

Fig. 1. Performance of teams

measure is the ratio between the number of matches won and the number of matches lost. So we did a performance study of some important cricket-playing countries (those that played more than 400 matches) in both form of cricket based on their win/loss ratio.

The performance of some selected Test playing countries from 1951 to 2009, One day playing counties from 1971 to 2009, and IPL playing teams from 2008 are shown in Fig. 1(a), Fig. 1(b) and Fig. 1(c) respectively. As clear from Fig. 1(a), Australia and West Indies are two countries whose win/loss ratio has crossed 4.5, while other countries have stayed below 2.0. For One day cricket, similar things can be observed in Fig. 1(b), Australia and West Indies have higher win/loss ratios than others. Some other interesting things can be seen in Fig. 11(a) like the dip in Australia's performance from 1950s to 1980s and then 
its rise back in 1990s and 2000s, West Indies showed an improvement in its performance during 1980s and the fell back during 1990s. Similarly, in Fig. 1(b), we can see some definite trends in countries like Australia, West Indies and South Africa, whereas the performance of India and Pakistan are mostly flat. It can be seen from Fig. 1(c) that Chennai Super Kings has won more than half the matches it played in all the six tournaments. Rajasthan Royals performed best in 2009 but then its performance has deteriorated after that. These observations encourage us to look at more details inside the cricket network.

\section{Cricket : A Complex Network}

In this section we discuss, how we can model cricket as a complex social network and relate complex network properties with the performance of the teams.

In order to get a deep understanding of performance of different teams in cricket according to runs scored in partnership, we have considered weighted undirected network models. In this model, each team is represented as a weighted network and each player in the team represents a node in that network. Two nodes (or players) are connected by an edge, if they have scored runs in partnership and the edge weight $w_{u, v}$ between two nodes $u$ and $v$ is given as

$$
w_{u, v}=\frac{\text { Total runs scored in partnership }}{\text { No. of Inns played together }}
$$

Table 1. Details of Test Cricket Network

\begin{tabular}{|c|c|c|c|c|}
\hline Property & AUS & WI & IND & PAK \\
\hline v & 102 & 98 & 86 & 62 \\
\hline e & 296 & 231 & 238 & 193 \\
\hline
\end{tabular}

Table 2. Details of One day Cricket Network

\begin{tabular}{|c|c|c|c|c|c|c|c|c|}
\hline Property & AUS & ENG & IND & NZ & PAK & SA & SL & WI \\
\hline v & 55 & 40 & 40 & 44 & 48 & 27 & 35 & 36 \\
\hline e & 122 & 88 & 115 & 115 & 134 & 81 & 94 & 116 \\
\hline
\end{tabular}

Table 3. Details of IPL Cricket Network

\begin{tabular}{|c|c|c|c|c|c|c|c|c|}
\hline Property & RCB & CSK & DC & DD & KingsXI & KKR & MI & RR \\
\hline v & 65 & 41 & 66 & 58 & 57 & 56 & 51 & 58 \\
\hline e & 226 & 142 & 253 & 235 & 236 & 207 & 224 & 251 \\
\hline
\end{tabular}

The main objective in this work is to view cricket from a social network perspective based on partnership, because partnership can be thought of as a 
social relationship, i.e., more the number of runs scored between two players in partnership, closer their social relationship. An undirected, weighted graph is created for each country. The details about the complexity of the Test network is given in Table-1. One day network in Table-2, and IPL in Table-3.

We have done all our experiments using Networkx [16], which is a Pythonbased package for creation, manipulation, and study of the structure, dynamics, and function of complex networks. Complex network structures have two basic set of properties, topological and structural. To characterize cricket as a complex network, we looked at the two set of properties for the cricket network.

Topological Characterization: Here we have considered degree and strength distribution of nodes. The resultant graph for the degree distribution for Test cricket network is shown in Fig. 2(a). Here $k$ is referred as degree and $P(k)$ denotes the degree distribution, i.e., probability that a given node is having degree $k$.

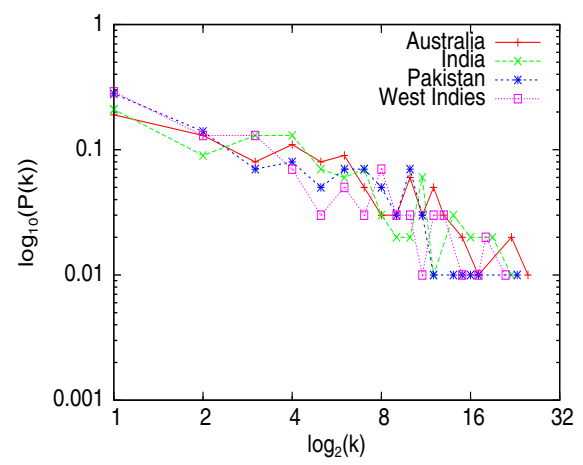

(a) For Test

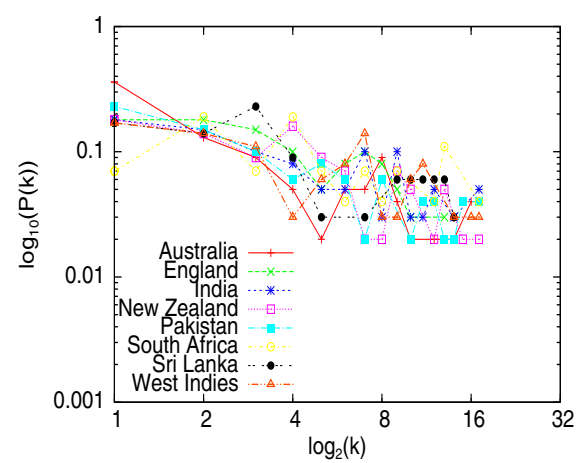

(b) For One day

Fig. 2. Degree Distribution

Here a degree of a node is determined by number of partners in it, i.e., number of team members with whom the node player has scored more than 30 runs in at least 3 innings. This distribution can be approximated by a power law behavior and the power law exponents (as described by Clauset et al. in [17]) for Australia, India, Pakistan and West Indies are found to be 3.50, 2.79, 2.89 and 2.37 respectively. Hence we can see a big difference in power law exponent between Australia and West Indies which reflects in their performance. Similar results are obtained for One day cricket (Fig. 2(b)). But, here degree of a node determines - number of partners with whom the player has scored more than 20 runs in at least 5 innings. This distribution also follows power law behavior. Australia has more players having smaller degrees as compared to other countries, which means a lot of Australian players are capable of creating partnership. So we can say that, Australia is not a few men team, which makes it a stronger 


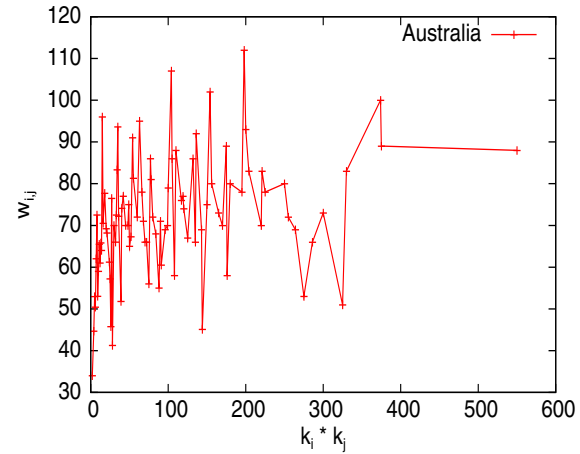

(a) For Test

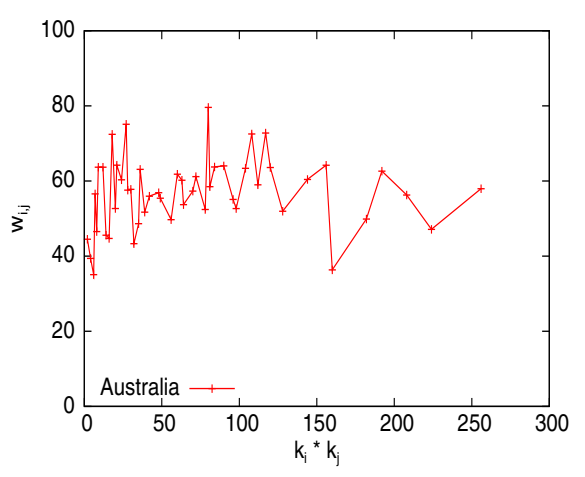

(b) For One-day

Fig. 3. Average weight as a function of end-point degrees for AUSTRALIA

team than others. But looking at the power-law exponents of all countries we found that, power law exponent for India is highest (3.50), while West Indies has the lowest (1.80), which may be one reason for the steady performances of India throughout and a heavy performance degradation of West Indies. We have also seen similar properties in the IPL network. We have not considered only weight of the edges in our analysis, because we found that there is almost no correlation between runs scored in partnerships and the popularity of the players for Australia network. Here popularity of the player means, the number of different partnerships a player is involved in, i.e., the degree of the player. We have observed this through the dependency analysis of weight between two nodes $i$ and $j, w_{i, j}$, with their corresponding degrees $k_{i}$ and $k_{j}$, where $k_{i}=\sum_{k} a_{i k}$ and $a_{i k}=1$, if player $i$ and $k$ scored runs partnership.

As we can see in Fig. 31(a), there is almost no correlation between edge weights and their end-point degrees except for higher $k_{i} * k_{j}$ values, are having higher $w_{i, j}$ values. But in ODIs Fig. [3 (b), most of the edge weights are between 50 to 70 regardless the values of $k_{i} * k_{j}$. For IPL also, most of the edge weights are between 15-30 irrespective for the end-point degrees. From these observations, we can conclude that, two higher degree players involve in higher partnership in Test cricket than One day cricket and T20, i.e., the role of popular (higher degree) players like RT Ponting, SR Waugh, AR Border are more significant in Test than One day and T20. In One day and T20 cricket almost all players have equal role to play in team performance.

Hence without considering the individual weight we consider group weights, which is the sum of all adjacent edges weight. So we looked at the extended definition of vertex degree $k_{i}$ in terms of vertex strength $s_{i}$, which is defined as

$$
s_{i}=\sum_{j=1}^{N} a_{i j} w_{i j}
$$




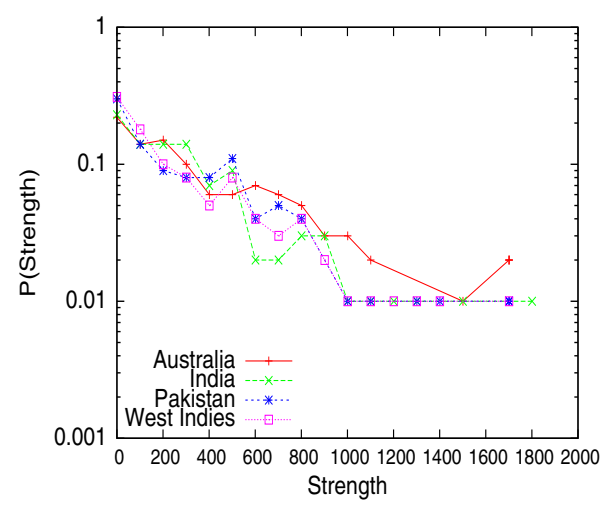

Fig. 4. Strength Distribution for Test Cricket

Fig. 4 shows the probability distribution $P(s)$ that a vertex has strength $s$ in the Test cricket. The functional behavior of this distribution is very similar to that of degree distribution $P(k)$ in Test and One day cricket. But while looking at the power law coefficient, we found that India has the minimum (1.63) power law coefficient. That may be reason due to which most of the time between 1950 to 2009, India's performance being least among all the four countries.

Structural Organization: In the cricket network, there may exist well connected groups of players - since two players who play for early wickets will be more connected to each other than those playing for first and the last wickets. In order to find the local group cohesiveness and correlations between the degree of connected vertices in the cricket network, we analyzed two social network characteristics clustering coefficient and assortativity.

For any node $v_{i}$, the clustering coefficient $c_{i}$ is defined as the fraction of connected neighbors of $v_{i}$ [6]. In general, this metric tells us how well a typical node's neighbors are connected in the network. The clustering coefficient of the network is the average clustering coefficient $C=N^{-1} \sum_{i} c_{i}$. Since the graphs involved here are weighted graph, we consider here the weighted clustering coefficient. In 11] this was defined as

$$
C_{i}=\frac{1}{s_{i}\left(k_{i}-1\right)} \sum_{j, h} \frac{\left(w_{i j}+w_{i h}\right)}{2} a_{i j} a_{i h} a_{j h}
$$

We have defined $C^{k}$ as average weighted clustering coefficient of all vertices having degree $k$. In all three different forms of cricket (Test, One day, and IPL), the networks show a decaying $C^{k}$. That means players with fewer partners usually make a well defined group in which all the players know each other. On the other hand, players with large degrees, know players from different groups, who in turn does not know each other. This is largely due to the partial change in the list of team players at certain times when a few old players are replaced with 


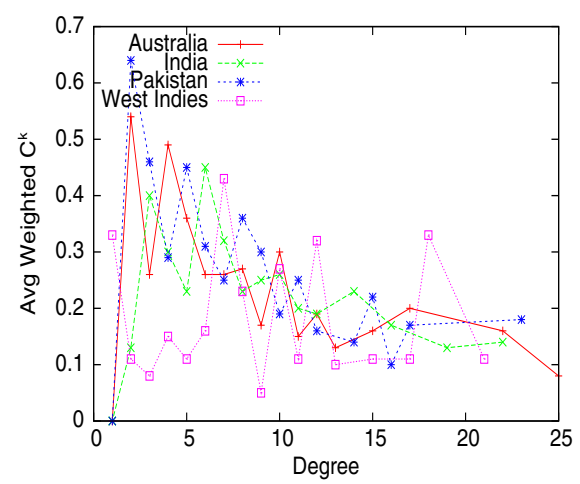

(a) For Test

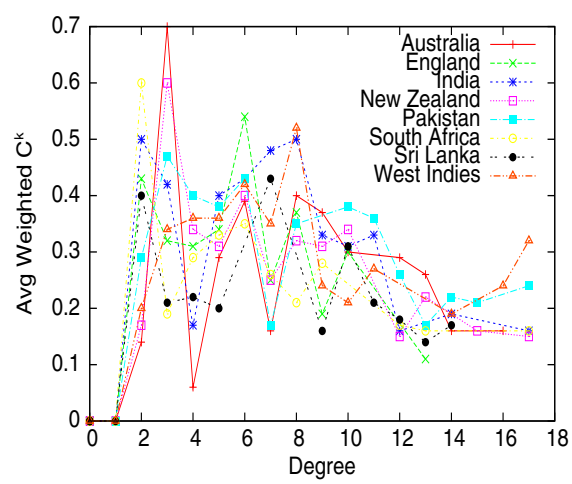

(b) For One day

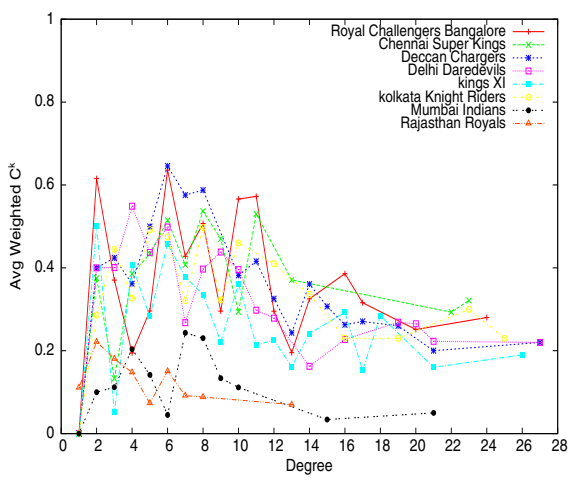

(c) For IPL

Fig. 5. Average weighted clustering coefficient as a function of $\mathrm{k}$

new players. The value average weighted $C^{k}$ is lower for Rajasthan Royals and Mumbai Indians as they have the larger number of partnerships with less number of players which can also be verified from Table-3 and is highest for Royal Challengers Bangalore.

To know the interconnection between nodes, we have looked at the average degree of nearest neighbors, $k_{n n}(k)$. This quantity is called as the joint degree distribution (JDD), which gives insight into the structure of the neighborhood of a node with degree $\mathrm{k}$. An increasing $k_{n n}(k)$ indicates a tendency of high degree nodes tend to connect to other high degree nodes. This property is popularly known as the assortative mixing 1819. A decreasing nature of this quantity defines disassortative mixing, where high-degree vertices have majority of lowdegree neighbors. Since the networks used are weighed networks, we consider the weighted average nearest-neighbor degree [11|18|19]. This is defined as 


$$
k_{n n, i}^{w}(k)=\frac{1}{s_{i}} \sum_{j=1}^{N} a_{i j} w_{i j} k_{j}
$$

If $k_{n n, i}^{w}(k)>k_{n n, i}(k)$ then edges with higher weights point to neighbors with larger degree and vice-versa. In both cases of Fig. [6(a) and (b), $k_{n n, i}^{w}(k)$ is plotted

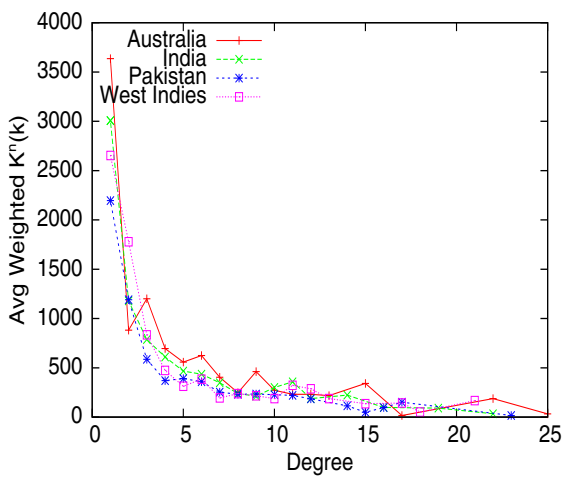

(a) For Test

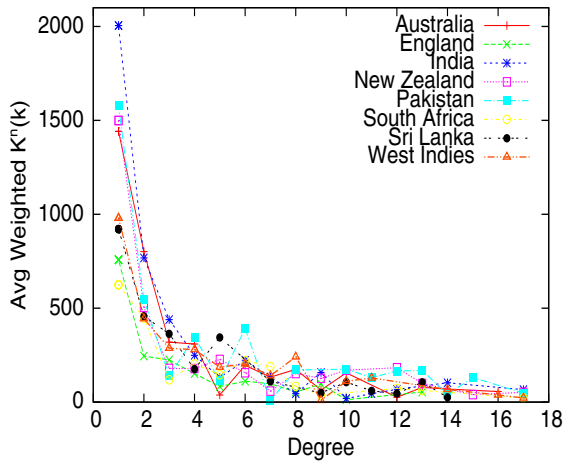

(b) For One day

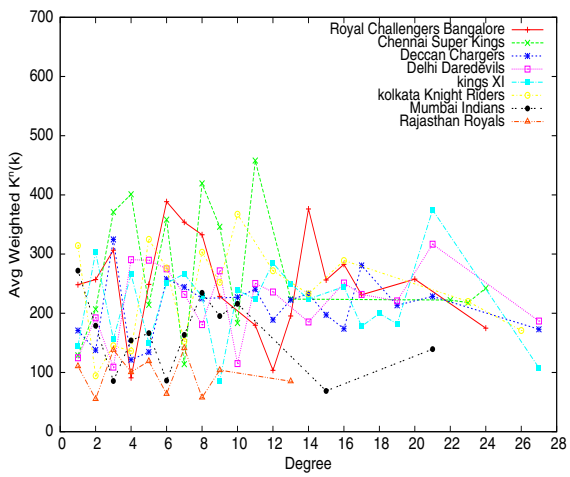

(c) For IPL

Fig. 6. Average weighted nearest-neighbor degree as a function of $\mathrm{k}$

as a function of $k$, which decays exponentially. This means the cricket network for the case of Test and One day cricket exhibit a disassortative mixing model in which players with higher degrees have majority of low-degree neighbors. That is the probability that two popular (higher degree) players score runs in partnership is less than that of one popular and one non-popular (lower degree) player. However, for the case of IPL (Fig. 6(c)), this coefficient does not decrease exponentially and the reason for this being that IPL inning is played only for 
twenty overs and thus it gives less chance to the lower degree players to do batting. From the above discussion now it is clear that, indeed cricket networks follow standard complex network characteristics.

\section{Observation from Cricket Network}

In this section we have discussed some important findings from our experiments to evaluate teams' performances. The properties of cricket networks are compared with the performances of teams as well as the format of the game.

\subsection{Performance vs. Network Characteristic}

We have shown that the degree and strength distribution follows power law in cricket networks, which means few players score, runs in partnership with lot of others players. To investigate further in this area, we have studied how the strength $s_{i}$ depends on the degree $k_{i}$. Therefore, the average strength $s(k)$ taken over $k$ was plotted against degree $k$ and we find that $s(k)$ increases linearly with degree $k$ as illustrated in the Fig. 7 for Test, One day and IPL. To support these experiments, randomized version of the networks were generated by random redistribution of the actual weights on the existing topology of the networks. Dependency of $s_{i}$ on $k_{i}$ for this random assignment of weights were compared with those for real-data. Result for Australia team are shown in Fig. 8, We can see that, curves for the real data and the randomized weights are very similar and well fit by an uncorrelated approximation $s(k)=\langle w\rangle k$, where $\langle w\rangle$ is the average weight in the network. This means the weights $w_{i j}$ are on average independent of $i$ and $j$. Only at very large degrees there is a slight departure from the expected linear behavior and the nodes' strength grows much faster with degree. So it does not matter who scores runs in partnerships, i.e., either the opening players or the tail-enders for the performance of the team. In Fig. 1(a), we can see that Australia and West Indies are the two countries who have shown significant rise and fall in their performances over the years. In order to enrich our findings, we want to study the evolution of performances in cricket over time. For that, we look at these two countries individually cross-sectioned over a period of 10 years and then see how the network properties have evolved over time. We have considered three basic measures: average degree $(\bar{k})$, average strength $(\bar{s})$ and clustering co-efficient (C) and try to figure out how these properties behave with time evolution. The mean connectivity $\bar{k}$ or average degree for the Australia and West Indies are shown in Fig. 9(a). The findings are very intuitive and interesting. We have observed that the average degree of the nodes follow similar behavior that of the performances of the corresponding countries shown in Fig. 1(a). Next we investigate if the average strength, $\bar{s}$, of nodes follow the same suit as the average degree or not. Fig. 9(b) shows the average strength of both the countries in different time periods. As expected, we find a similar trend here as well. The behavior here is closer to the performance trends as compared to average degree. Lastly we try to find out the trends of evolution for average 


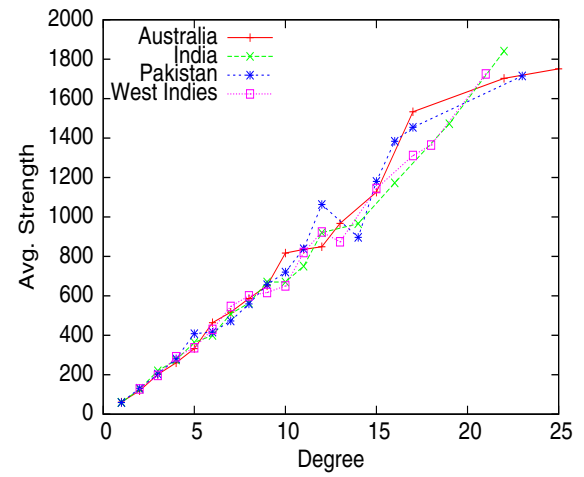

(a) For Test

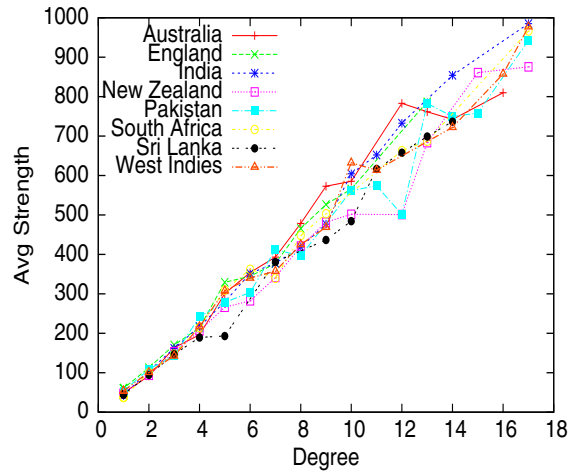

(b) For One day

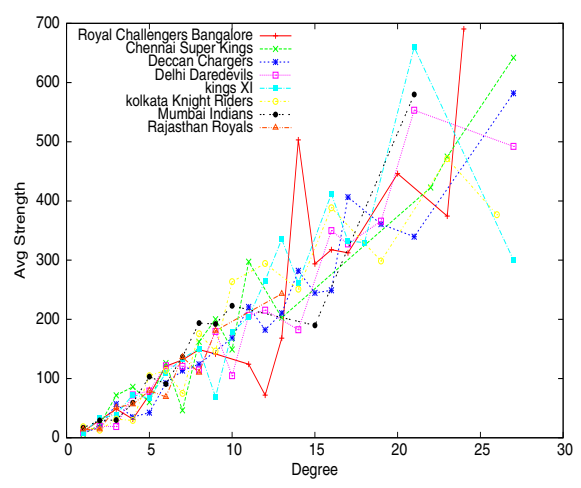

(c) For IPL

Fig. 7. Strength vs. Degree

clustering coefficient. Same behavior is shown in this case also. As clear from Fig. 9(c), we can see the striking similarities between team's performance and its average clustering coefficient over time.

\subsection{Formats of the Game vs. Network Characteristics}

Here we discuss some interesting findings related to the difference between Test, ODI and T20 cricket using complex network properties. In Fig. 4, we can clearly see that, Australia has more players having smaller degrees compared to other countries in One day cricket, which is not seen in Test Cricket. It follows that; a lot of players are capable of creating partnership for Australia in One day cricket. This probably makes Australia a stronger team in ODIs. Another interesting 


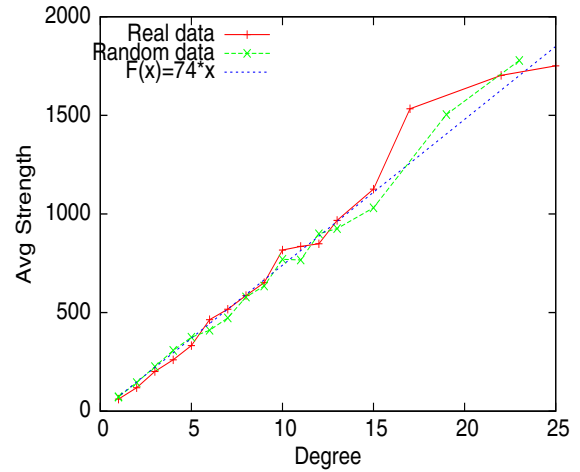

(a) For Test

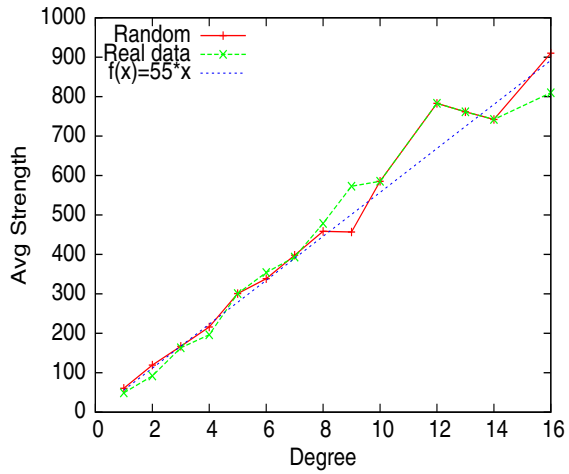

(b) For One day

Fig. 8. Comparison between real data and randomized weights for Australia

thing can be seen in Fig. 4, the power law coefficient for India is maximum among all countries, that makes a strong reasoning about the consistently improved performance of India team in One day cricket. From Fig. 4(b), it can be observed that the relationship between end points degrees and their respective weight do not vary much as compared to the Test cricket. This implies the role of the popular batsmen is more significant in Test cricket than the One day cricket. In One day cricket almost all players have equal role to play in team performance. As we did time evolution experiment for Test cricket, we have also performed the similar experiments for the One day cricket and IPL. As we know from Fig. 1(b) that, the performances of Australia, West Indies and South Africa are very fluctuating, we have looked into these three countries individually crosssectioned over a period of 5 years and then shown how the network properties have evolved over time. The results are given in Fig. 10, But here the network properties like degree, strength and clustering coefficient do not follow the same trend as the performance of the team. But the number of nodes and edges increase as the performance of the team increases. From this we can conclude that the partnership plays more crucial role in Test cricket than in One day cricket.

In the case of IPL the average strength, Fig. 11(b), follows the pattern similar to the performance of the team. The evolution of average degree and clustering coefficient with time does not follow the same trend as the performance of the team. The reason for this can be explained as, some of the players played for different franchises in different seasons and there are lot of changes made in the batting order. The change in batting order matters a lot in case of IPL as it being a T20, only 20 overs are played and hence not all players get a chance to do batting. 


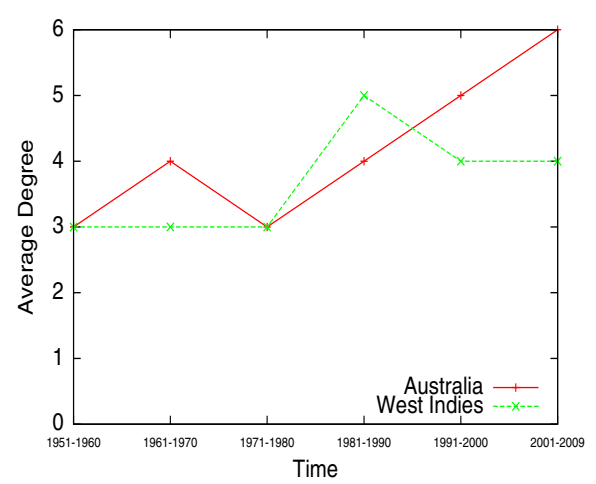

(a) Degree Evolution

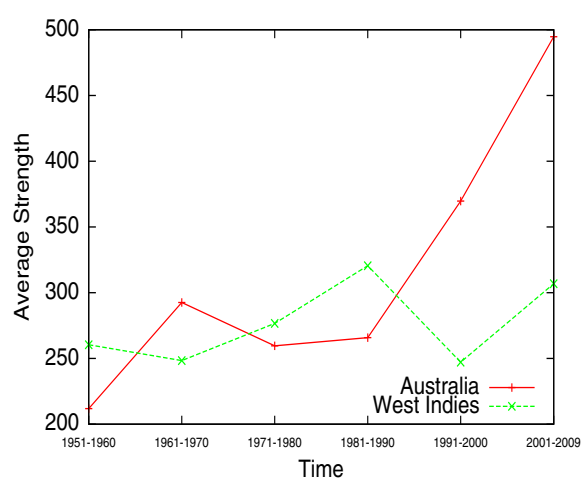

(b) Strength Evolution

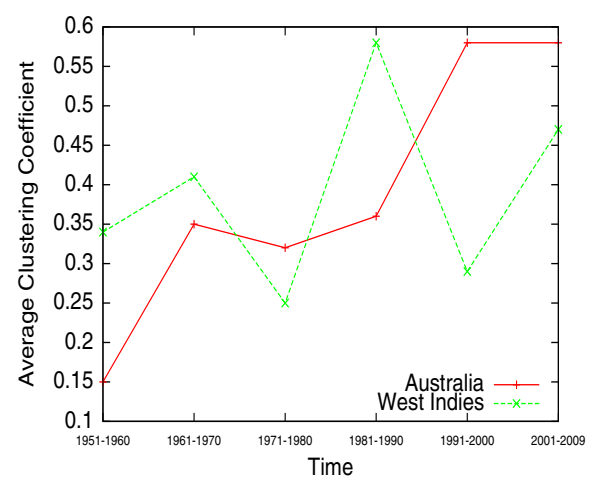

(c) Clustering Coefficient Evolution

Fig. 9. Time Evolution of Australia and West Indies in Test

\section{Conclusion}

In this work, we have modeled cricket as a complex social network, by showing that degree distributions and strength distributions follow power-law and also decrease in clustering coefficient with degree and having disassortative properties. We also found few hubs in the networks; interestingly these hubs in the networks are the popular batsmen of their respective countries like Sachin Tendulkar and Ricky Ponting. We have also analyzed the cricket network with respect to the performance of teams in all the three formats of cricket, Test, ODIs and T20. We found that, the edge weights are largely independent of end-points degree, i.e., there is almost no correlation between the partnership runs scored and the pair of partners, except the case for very low-degree players who in general score 


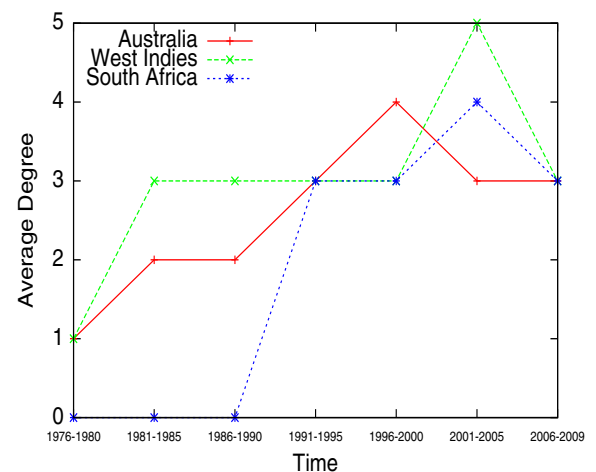

(a) Degree Evolution

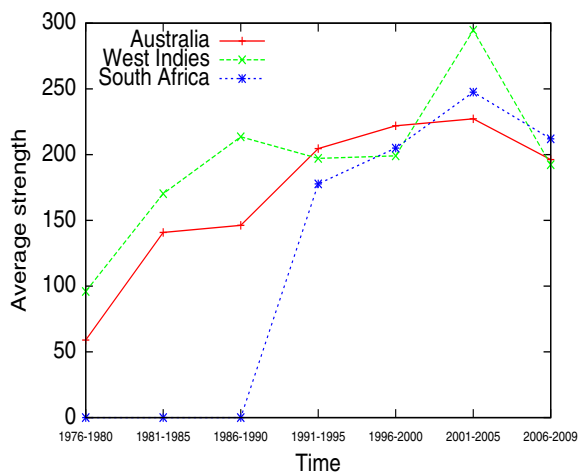

(b) Strength Evolution

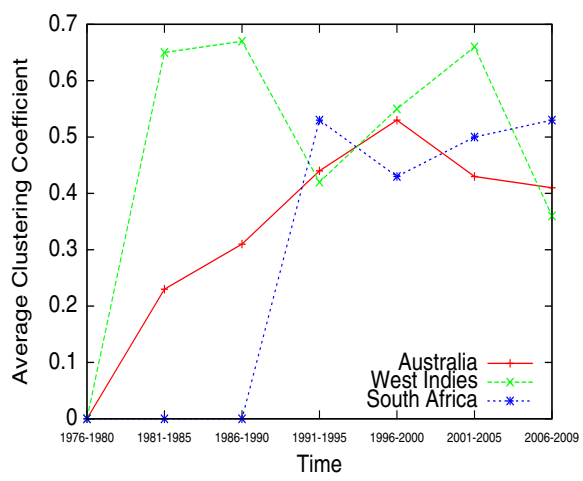

(c) Clustering Coefficient Evolution

Fig. 10. Time Evolution of Australia, West Indies and South Africa in One day

less and very large-degree players score more in Test cricket. Whereas in ODIs and T20, edge weights mostly have a constant relationship with end-point degrees. Hence in Test cricket, larger degree players like Sachin Tendulkar, Rahul Dravid are more significant than others but in One day cricket and IPL almost all players are equally important to the team. We have shown that, the clustering coefficient of nodes decrease in the cricket networks. Hence hubs in the networks have very dispersed neighborhoods while the low-degree vertices form well-connected groups. This behavior is explained as the larger-degree players partner with players who were on the team in different time periods. We have shown that, the average degrees of nearest neighbors of nodes with degree $k$ decays exponentially with $k$ for Test and ODIs, which signifies a disassortative behavior of the network, but generally real social networks satisfy assortative behavior. So in cricket network, high degree nodes have majority of neighbors with low degrees, whereas opposite holds for low-degree vertices. Interestingly 


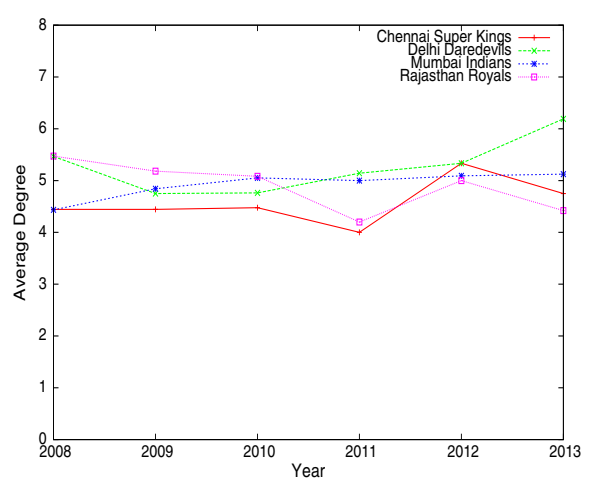

(a) Degree Evolution

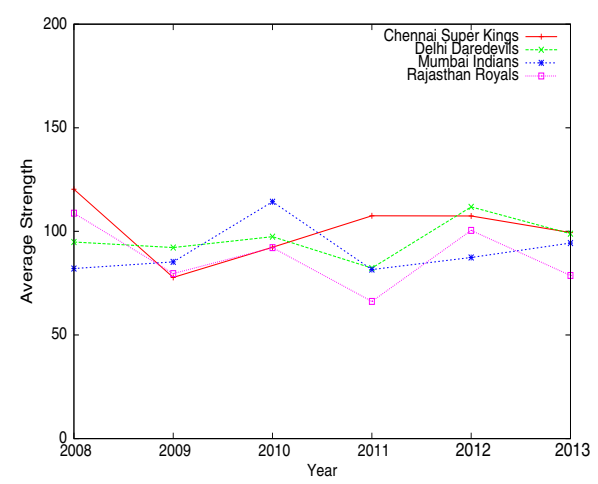

(b) Strength Evolution

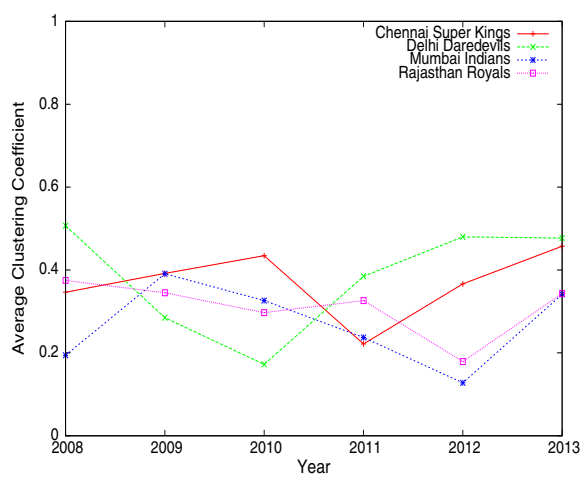

(c) Clustering Coefficient Evolution

Fig. 11. Time Evolution in IPL

we found similar trend follows in all forms of cricket. Due to large difference in the performance of Australia and West Indies in Test matches, we have observed the performance of teams with time evolution. We get very surprising results as we see that the average degree and the clustering coefficient follow the same trend as the performance of the country for Test cricket. But while doing the similar experiment for One day and T20 cricket, we have got completely opposite results, i.e., these properties are no way related to the performance of the team. Hence, the most important conclusion is, partnership plays more significant role in Test matches than in One day and T20 matches. As future works, one can go further in the time evolution of network characteristics. As these properties are directly correlated to the teams performance, one can think for developing models in this frame-work to predict the match outcomes. 


\section{References}

1. Albert, R., Jeong, H., Barabasi, A.L.: The diameter of the world wide web. Nature 401, 130 (1999)

2. Ahn, Y., Han, S., Kwak, H., Moon, S., Jeong, H.: Analysis of topological characteristics of huge online social networking services. In: WWW 2007: Proceedings of the 16th International Conference on World Wide Web, pp. 835-844. ACM, New York (2007)

3. Newman, M.E.: The structure and function of complex networks. SIAM Review 45, $167(2003)$

4. Newman, M.E.: The structure of scientific collaboration networks. Proceedings of the National Academy of Sciences 98(2), 404-409 (2001)

5. Faloutsos, M., Faloutsos, P., Faloutsos, C.: On power-law relationships of the internet topology. In: SIGCOMM 1999: Proceedings of the Conference on Applications, Technologies, Architectures, and Protocols for Computer Communication, pp. 251-262. ACM, New York (1999)

6. Watts, D.J., Strogatz, S.H.: Collective dynamics of 'small-world' networks. Nature 393(6684), 440-442 (1998)

7. Milgram, S.: The small world problem. Psychology Today 2, 60-67 (1967)

8. ESPNCricinfo: Cricket website, http://www.espncricinfo.com/ (accessed 2013)

9. Boccaletti, S., Latora, V., Moreno, Y., Chavez, M., Hwang, D.U.: Complex networks: Structure and dynamics. Physics Reports 424(4-5), 175-308 (2006)

10. Newman, M.E.: Analysis of weighted networks. Physical Review E 70, 56-131 (2004)

11. Barrat, A., Barthelemy, M., Pastor-Satorras, R., Vespignani, A.: The architecture of complex weighted networks. Proceedings of the National Academy of Sciences 101, 37-47 (2004)

12. De Melo, P.O.V., Almeida, V.A., Loureiro, A.A.: Can complex network metrics predict the behavior of NBA teams? In: KDD 2008: Proceeding of the 14th ACM SIGKDD International Conference on Knowledge Discovery and Data Mining, pp. 695-703. ACM, New York (2008)

13. Onody, R.N., De Castro, P.A.: Complex network study of Brazilian soccer players. Physical Review E 70, 37-103 (2004)

14. Damodaran, U.: Stochastic dominance and analysis of ODI batting performance: The Indian cricket team, 1989-2005. Journal of Sports Science and Medicine 5(4), 503-508 (2006)

15. Allsopp, P.E., Clarke, S.R.: Rating teams and analysing outcomes in One-day and Test cricket. Journal of The Royal Statistical Society Series A 167(4), 657-667 (2004)

16. NetworkX: Networkx documentation, http://www.networkx.lanl.gov/ (accessed 2013)

17. Clauset, A., Shalizi, C.R., Newman, M.E.: Power-law distributions in empirical data. SIAM Review 51(4) (November 2009)

18. Leung, C.C., Chau, H.F.: Weighted assortative and disassortative networks model (2006)

19. Chang, H., Su, B., Zhou, Y., He, D.: Assortativity and act degree distribution of some collaboration networks. Physica A: Statistical Mechanics and its Applications 383(2), 687-702 (2007) 